\title{
REFLECTIVITY SPECTRA OF CdTe IMPLANTED AT ROOM AND LIQUID NITROGEN TEMPERATURE
}

\author{
E. Czarnecka-Such, A. Kisiel, A. RodziK \\ Institute of Physics, Jagiellonian University \\ Reymonta 4, 30-059 Kraków, Poland \\ AND Z. GolACKI \\ Institute of Physics, Polish Academy of Sciences \\ Al. Lotników 32/46, 02-668 Warszawa, Poland
}

\begin{abstract}
The fundamental reflectivity spectra of monocrystalline CdTe, implanted with $\mathrm{Ag}$ ions at room temperature and with $\mathrm{Er}$ ions at liquid nitrogen temperature, are investigated in the $0.5-6.0 \mathrm{eV}$ energy range. The analysis of the obtained spectra leads to the suggestion that temperature of implantation influences the obtained results much more decisively than values of other parameters. For the implantation carried out at $300 \mathrm{~K}$ no significant changes in reflectivity spectra are observed regardless of magnitude of the ion dose (up to $5 \times 10^{15} / \mathrm{cm}^{2}$ ) and this fact, in our opinion, is due to the self-annealing effect. For samples implanted at temperature $77 \mathrm{~K}$ with comparable doses of ions, however, the characteristic changes of shape and intensity of reflection coefficient spectra appear. The manner of this changes gives evidence that temperature $77 \mathrm{~K}$ is low enough to make the radiation induced lattice defects stable (frozen-in) which are responsible for the observed behaviour of CdTe fundamental reflectivity spectra.
\end{abstract}

PACS numbers: $61.80 . \mathrm{Jh}, 61.70 . \mathrm{Tm}, 78.20 . \mathrm{Ci}$

\section{Introduction}

The analysis of fundamental reflectivity spectra is a widely used method to study electron structure of semiconductors [1]. Because of the fact that the band structure of a material is directly related to its crystalline structure (the existence of translational symmetry, by distance of at least several nearest neighbours, is necessary), it is possible to use this technique to obtain qualitative information about lattice disorder produced by ion implantation [2]. Moreover, this method, by possibility of choosing appropriate parameters of implantation (kind of ions, its mass, fluence, energy, etc.), allows to investigate the intermediate states describing the transformation from crystalline to amorphous-like state by a gradual change of defect concentration [3]. 


\section{Experiment}

The optical reflectivity spectra of CdTe were investigated in the $0.5-6.0 \mathrm{eV}$ spectral energy range for mechanically polished and chemically etched samples, using an automatic two-beam spectrometer controlled by a computer [4]. Room temperature ion implantation was carried out in the Institute of Nuclear Physics in Kraków with $70 \mathrm{keV} \mathrm{Ag}$ ions (doses in the range $8 \times 10^{12} / \mathrm{cm}^{2}-5 \times 10^{15} / \mathrm{cm}^{2}$ ). The other series of implantations was performed at liquid nitrogen temperature with $100 \mathrm{keV}$ Er ions in the Institute of Physics of Marie Curie-Skłodowska University in Lublin, with dose $2 \times 10^{14} / \mathrm{cm}^{2}, 1 \times 10^{15} / \mathrm{cm}^{2}$ and $5 \times 10^{15} / \mathrm{cm}^{2}$. In the above mentioned energy range (area of fundamental absorption) the reflectivity spectra contain information about the CdTe layer with the thickness not greater than $500 \AA$, due to high absorption coefficient in the considered spectral range. At the same time, the ions with the energy of $\approx 70-100 \mathrm{keV}$ produce the defects in the layers of a comparable and even greater thickness. Thus, the reflectivity spectra in this range give the information only about the implanted layer.

\section{Results and discussion}

The results of reflectivity measurements in the $0.5-6.0 \mathrm{eV}$ energy range for $\mathrm{CdTe}$ samples implanted at room temperature with various doses of $70 \mathrm{keV} \mathrm{Ag}$ ions are shown in Fig. 1. The salient feature of the presented spectra is the appearance of three distinguished maxima connected with CdTe band structure: $E_{1}, E_{1}+\Delta_{1}$ and $E_{2}$, and a small one, $E_{0}$, related to band gap transition. The spectra shown in Fig. 1 are qualitatively similar, any systematic changes of their intensity or shape are not observed. The measurable shifts of the maxima energies do not appear either. This lack of significant and meaningful modification of intensity and shape of the fundamental reflectivity spectra is tantamount to the absence of weighty changes of lattice order in the studied samples. It should be emphasized that, in the case when implantation is carried out at room temperature, even for such great dose of $70 \mathrm{keV} \mathrm{Ag}$ ions as $5 \times 10^{15} / \mathrm{cm}^{2}$, the density of radiation induced lattice defect is not enough to influence the mean features of $\mathrm{CdTe}$ fundamental reflectivity spectra. Reflectivity coefficient spectra for CdTe implanted with $100 \mathrm{keV} \mathrm{Er}$ ions at the liquid nitrogen temperature are presented in Fig. 2. The main feature of these spectra is that optical reflectivity peaks $E_{1}, E_{1}+\Delta_{1}$, and $E_{2}$ decrease smoothly with increasing ion dose and finally disappear (except $E_{0}$ ) when a certain critical fluence of $E r$ ions is reached (dose approximately $5 \times 10^{15} / \mathrm{cm}^{2}$ ). This characteristic behaviour of reflectivity spectra can be considered as a consequence of gradual disordering of originally crystalline $\mathrm{CdTe}$ sample as a result of implantations. More significant drop of reflection coefficient intensity at higher energies $\left(E_{2}\right.$ maximum) than at infrared energy range can be related to the increase in roughness on samples surface.

Such a fundamental inconsistency between results obtained in described above two series must be, in our opinion, caused by essential difference of implantation temperature in both cases (77 and $300 \mathrm{~K})$ [5]. 


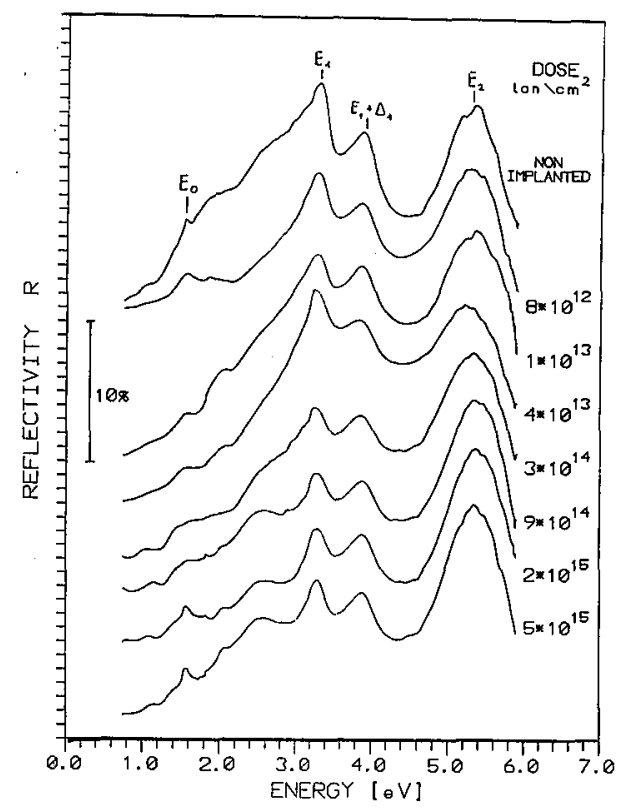

Fig. 1. Fundamental reflectivity spectra for CdTe samples implanted with Ag ions at room temperature in $0.5-6.0$ energy range.

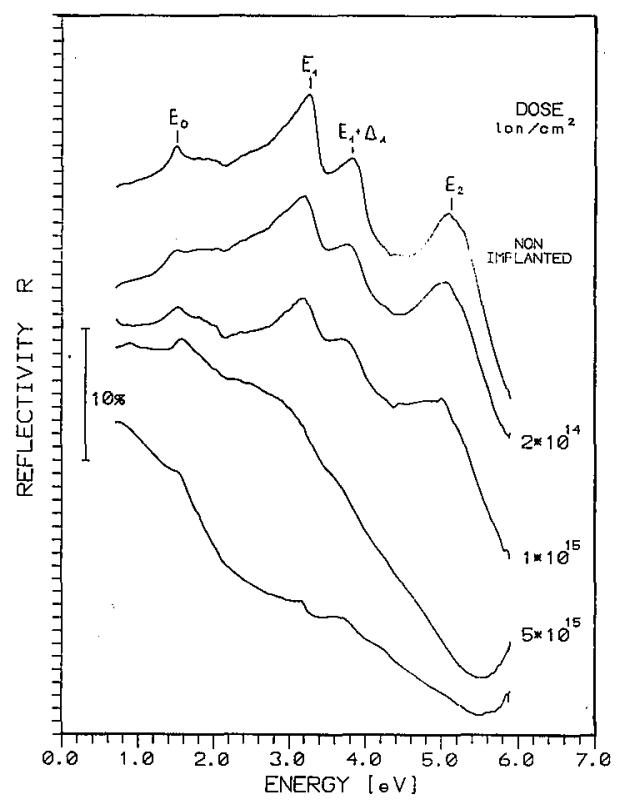

Fig. 2. Fundamental reflectivity spectra for CdTe samples implanted with Er ions at liquid nitrogen temperature in 0.5-6.0 energy range; the lowest curve is for the implanted sample heated after implantation with the use of laser. 


\section{References}

[1] J.C. Phillips, Solid State Phys. 18, 55 (1966).

[2] E. Czarnecka-Such, A. Kisiel, Surf. Sci. 193, 221 (1988).

[3] J.F. Zigler, J.P. Biersack, V. Littmark, The Stopping and Range of Ions in Solids, Vol. 1, Pergamon Press, New York 1985.

[4] J. Oleszkiewicz, M. Podgórny, A. Kisiel, W. Knapik, Opt. Appl. 15, 163 (1985).

[5] K. Zanio, Semiconductors and Semimetals, Vol. 13, Academic Press, New York 1978. 